\title{
Allergieforschung: Suche nach neuen Ansätzen
}

D ie allergologische Lifestyle-Forschung hat ein neues Highlight: Anthroposophische Lebensweise schützt vor Allergien. Dies ist das Ergebnis einer Untersuchung bei 295 schwedischen Kindern zwischen dem 5. und 13. Lebensjahr. Diese litten seltener an Asthma, atopischem Ekzem oder Rhinokonjunktivitis und hatten weniger Sensibilisierungen gegen häufige Aero- und Nahrungsmittelallergene als 380 Kontrollkinder (Hauttest, Spezifisches IgE). Eine besonders strikte Beachtung anthroposophischer Lebensweise reduzierte das Risiko deutlich (Odds Ratio 0,56).

Welche der Besonderheiten anthroposophischer Lebensweise für diese erstaunliche Reduktion des Atopierisikos verantwortlich sind, kann von der Studie nicht beantwortet werden. Signifikante Verringerungen bzw. Trends wurden gezeigt für Stillen ohne Beikost über mindestens 4 Monate, strikte Vermeidung von Antibiotika, fiebersenkenden Mitteln, Impfungen, fermentiertem Gemüse, Masern bzw. die vorwiegende Ernährung mit biodynamischer Kost (Alm et al., Lancet 1999; 353: 1485-8).

Diese Arbeit wird sicherlich die Forschung über die Ursachen der steigenden Allergieprävalenz zusätzlich beflügeln - zahlreiche Modelle der Analyse von Bevölkerungsgruppen mit spezieller Lebensweise sind vorstellbar. Die Vielzahl der Einflußfaktoren erschwert die Planung epidemiologischer Studien. So erfährt man z.B. in der o.g. Studie nichts über die Familiengröße bzw. Geschwisterzahl der Kinder, Angaben über die häusliche und schulische Allergenbelastung werden ebenfalls nicht gemacht. Wir sind also immer noch weit von einer Aufklärung der kausalen Zusammenhänge entfernt - und die Zeit drängt.

Neue Forschungsansätze sind willkommen. Bemerkenswert in diesem Zusammenhang ist eine vor kurzem publizierte Arbeit (mit Editorial) über die heilende Wirkung von Worten bei Asthmapatienten (Smyth et al. JAMA 1999; 281: 1304-9, Kommentar 1328). Die Autoren konnten zeigen, daß das bloße Schreiben über vergangene streßreiche Erlebnisse zu einer Verringerung der Asthmasymptomatik führt. 39 Probanden wurden aufgefordert, über das ,streßreichste Ergebnis ihres Lebens" innerhalb von 20 Minuten an drei konsekutiven Tagen zu schreiben. 22 asthmakranke Kontrollprobanden notierten lediglich ihren Tagesablauf. Vier Monate später hatten die schriftlich ,entlasteten“ Probanden eine signifikant gebesserte Lungenfunktion (FEV1: 63,9 auf 76,3\%, Kontrollen 64,0 auf $65,3 \%$ ).

Die Ergebnisse sind geeignet, die Forschung über die Zusammenhänge zwischen Neuropsychologie, Immunologie und Atopie mit Nachdruck zu fördern - auch diese Faktoren beeinflussen möglicherweise das Atopierisiko des „Western Lifestyle“.

Die Allergieforschung hierzulande hat einen wesentlichen Beitrag zur Allergie-Epidemiologie geleistet, auch im internationalen Maßstab. Unter der irreführenden Überschrift „Allergieforschung: Deutschland hat Defizite“" wurde in Heft 3/1999 von Bild der Wissenschaft herausgestellt, daß die Zahl deutscher Publikationen auf dem Allergiesektor im internationalen Vergleich hinter der der USA und Großbritanniens, aber noch vor der Frankreichs und Japans liegt. Innerhalb Deutschlands rangiert in der Zahl allergologischen Publikationen der Universitäten von Berlin vor der der Universitäten von München, sowie den Uni-

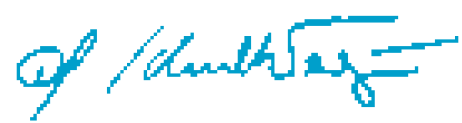

Prof. Dr. G. Schultze-Werninghaus versitäten Hannover, Bochum, Jena und Freiburg.

Nach Zitaten lagen die Universitäten von Berlin vor München, Bochum, Freiburg, Hannover und Münster. Als herausragend werden unter den Wissenschaftlern erwähnt: Prof. Wahn, Berlin; unter den führenden Institutionen werden genannt: das RudolfVirchow-Klinikum, Charité Berlin, das Forschungszentrum für Umwelt und Gesundheit, Neuherberg und München, das Robert-Koch-Institut, Berlin, sowie - mit besonderer Herausstellung - das Berufsgenossenschaftliche Forschungsinstitut für Arbeitsmedizin, Bochum. Zu ergänzen wäre die Technische Universität München, die - im Gegensatz zu den Berliner Universitäten - nicht mit der LMU München zusammengefaßt wird, und dennoch an 6 . bzw. 9. Stelle der Bewertungsskalen liegt. Unter den pharmazeutischen Firmen werden die publizistischen Aktivitäten der Firma Allergopharma, Reinbek, herausgestellt.

Dies ist nur ein Meinungsbild, das sicherlich kritisch gewürdigt werden muß. Wir sollten uns aber freuen über eine Reihe aktiver Arbeitsgruppen und eine international gestärkte Position gemessen an Quantität und Qualität der Publikationen.

Wir hoffen, Sie auf der Tagung der Deutschen Gesellschaft für Allergologie und klinische Immunologie vom 20.-24. Oktober 1999 in München begrüßen zu können. Das Thema der 21. Tagung an der Schwelle zum 21. Jahrhundert: „Allergie 2000: Probleme, Strategien und praktische Konsequenzen".

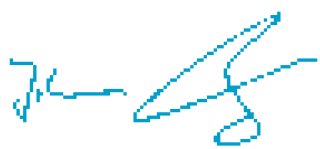

Prof. Dr. Dr. J. Ring 